\title{
Clinical effect of atorvastatin-trimetazidine combined treatment of coronary heart disease.
}

\author{
Ji-Dong Song, Li-Li Wang* \\ Department of the First Heart Surgery, Tangshan Workers' Hospital, Tangshan, Hebei, PR China
}

\begin{abstract}
Objective: To explore clinical effect of atorvastatin-trimetazidine combined treatment of patients with coronary heart diseases.

Methodology: A total of 98 patients with coronary heart diseases accepted by our hospital from February 2016 to June 2017 were divided into two groups according to random principle, namely reference group $(n=49)$ and study group $(n=49)$. Patients in the reference group accepted atorvastatin treatment while those in the study group accepted atorvastatin-trimetazidine combined treatment. Then, a statistical comparison in the aspects of clinical effect, blood lipid index, cardiac function index, and occurrence of adverse reactions between the two groups was carried out.

Results: Overall effective rate of patients in the study group is $\mathbf{9 3 . 8 8 \%}$, which is significantly different from that of patients in the reference group $(\mathbf{7 9 . 5 9 \%})(\mathbf{P}<\mathbf{0 . 0 5})$. Triglyceride (TG), Total Cholesterol (TC), Low-Density Lipoprotein Cholesterol (LDL-C) and High-Density Lipoprotein Cholesterol (HDLC) are significantly lower than those of patients in the reference group $(P<0.05)$. Left Ventricular Ejection Fractions (LVEF) of patients in the study group were significantly larger than those of patients in the reference group $(P<0.0)$ while their Left Ventricular End-Systolic Dimension (LVESD) and Left Ventricular End-Diastolic Dimension (LVEDD) are both smaller than those of patients in the reference group $(\mathbf{P}<\mathbf{0 . 0 5})$.

Conclusion: Clinical effect of atorvastatin-trimetazidine combined treatment on patients with coronary heart diseases is prominent and can effectively improve blood lipid level and cardiac functions of patients. Such combination is a therapeutic schedule deserving clinical promotion and application because it is safe and reliable.
\end{abstract}

Keywords: Coronary heart disease, Atorvastatin, Trimetazidine, Clinical effect.

Accepted on November 29, 2017

\section{Introduction}

Coronary heart disease, which is also known as coronary atherosclerotic heart disease, refers to a heart disease featuring myocardial anoxia, ischemia or necrosis due to blocked or narrowed vessel lumen cause by atherosclerotic lesion, and clinical manifestations of such disease mainly include palpitation, chest congestion, and angina $[1,2]$. In recent years, with increasing morbidity, coronary heart disease is most evident in middle-aged and elderly people and seriously threatens life and health of patients. As commonly used drugs for the clinical treatment of coronary heart diseases, atorvastatin and trimetazidine have effects of antiinflammation, lipid reduction, blood vessel protection, cardiac function recovery, and plaque dissolution facilitation with favorable clinical application effect [3,4]. In this study, 98 patients with coronary heart diseases accepted by our hospital from February 2016 to June 2017 were studied to analyse the clinical effect of atorvastatin-trimetazidine combined treatment.

\section{Materials and Methodology}

\section{General information}

A total of 98 patients with coronary heart diseases accepted by our hospital from February 2016 to June 2017 were divided into two groups according to random principle, namely reference group $(n=49)$ and study group $(n=49)$. Inclusion criteria: (1) All patients met diagnostic criteria for coronary heart diseases in naming and diagnostic criteria of ischemic heart diseases. (2) Patients could positively cooperate in completing the study with clear consciousness but no communication disorders. (3) Patients voluntarily participated in this study and signed an informed consent form. Exclusion criteria: (1) IV cardiac functional grade accompanied by serious cardiac failure; (2) concurrent with serious hepatic and renal dysfunction; (3) serious infection and tumor; (4) allergy to therapeutic drugs. The reference group comprised 23 females and 26 males, of which the youngest was $40 \mathrm{y}$ old and the oldest was $81 \mathrm{y}$ old, with average age being $(60.92 \pm 6.31 \mathrm{y}$ old). The shortest course of the disease was one year while the 
longest was five years, with an average course of the disease being $(2.72 \pm 0.55 \mathrm{y})$. SPSS22.0 software was used to process the preceding information of patients in the two groups, and no significant difference existed in inter-group comparison $(\mathrm{P}>0.05)$.

\section{Methodology}

Patients in both groups were provided with symptomatic treatments, such as drugs for vascular dilation and diuretic. On this basis, patients in the reference group were treated with atorvastatin (Zhejiang Neo-Dankong Pharmaceutical Co., Ltd, SFDA approval number: H20133127), and oral administration continuously lasted for two weeks once per day and $10 \mathrm{mg}$ once. Patients in the study group accepted atorvastatintrimetazidine combined treatment (Beijing NHU Pharmaceutical Co., Ltd, SFDA approval number: H2006516). Atorvastatin treatment was consistent with that in the reference group, and oral administration of trimetazidine was conducted three times per day, with $20 \mathrm{mg} / \mathrm{d}$ dosage continuously for two weeks.

\section{Observational indexes}

Statistical comparison between the two groups in the aspects of clinical effect, blood lipid index, and cardiac function index, as well as the occurrence of adverse reactions, was conducted. Blood lipid indexes mainly include TG, TC, LDL-C, and HDLC. Cardiac functional indexes comprise LVEF, LVESD, and LVEDD. Adverse reactions are emesis and insomnia.

\section{Criteria for clinical effects}

Excellent: After treatment, clinical symptoms of patients disappeared, T-wave inversion and shallowing were larger than $50 \%$, and ST-segment elevation was larger than $0.15 \mathrm{mV}$.

Effective: After treatment, clinical symptoms of patients were slightly alleviated, and $\mathrm{T}$ waves and ST segments were improved to different degrees.

Ineffective: After treatment, patients failed to reach the preceding criteria mentioned. The sum of excellent and effective rates was referred to as total effective rate.

\section{Statistical analysis}

SPSS22.0 software was used to process observed data of patients in the two groups. Numeration data (total clinical effective rate and occurrence rate of adverse reactions) were expressed in the form of percentage with $\chi^{2}$ test while measurement data (TG, TC, LDL-C, HDL-C, LVEF, LVESD, and LVEDD) were expressed in the form of $(\overline{\mathrm{x}} \pm \mathrm{s})$ with $\mathrm{t}$-test. If $\mathrm{P}<0.05$, then the comparative difference between the two groups is significant.

Table 1. Comparative analysis of clinical effects between the two groups $(n(\%))$.

\begin{tabular}{llll}
\hline Group Excellent $\quad$ Effective Ineffective & $\begin{array}{l}\text { Total } \\
\text { effective rate }\end{array}$ \\
\hline
\end{tabular}

\begin{tabular}{lllll}
\hline $\begin{array}{l}\text { Study group } \\
(\mathrm{n}=49)\end{array}$ & $10(20.41)$ & $36(73.47)$ & $3(6.12)$ & $46(93.88)$ \\
\hline $\begin{array}{l}\text { Reference } \\
\text { group }(\mathrm{n}=49)\end{array}$ & $5(10.20)$ & $34(69.39)$ & $10(20.41)$ & $39(79.59)$ \\
\hline$X^{2}$ & & 4.3457 \\
\hline$P$ & & 0.0371 \\
\hline
\end{tabular}

\section{Results}

\section{Comparative analysis of curative effects between the two groups}

The total effective rate of patients in the study group is $93.88 \%$, which is significantly different from that of patients in the reference group $(79.59 \%)(\mathrm{P}<0.05)$, as shown in Table 1 .

\section{Comparative analysis of blood lipid indexes between the two groups}

TG, TC, LDL-C, and HDL-C of patients in the study group are all significantly lower than those in the reference group $(\mathrm{P}<0.05)$, as shown in Table 2 .

Table 2. Comparative analysis of blood lipid indexes between the two groups $(\bar{x} \pm s, \mathrm{mmol} / \mathrm{L})$.

\begin{tabular}{|c|c|c|c|c|}
\hline Group & TG & TC & LDL-C & HDL-C \\
\hline $\begin{array}{l}\text { Study group } \\
(n=49)\end{array}$ & $1.35 \pm 0.31$ & $2.23 \pm 0.20$ & $1.66 \pm 0.23$ & $5.62 \pm 2.56$ \\
\hline $\begin{array}{l}\text { Reference } \\
\text { group }(n=49)\end{array}$ & $1.89 \pm 0.36$ & $3.18 \pm 0.22$ & $1.93 \pm 0.26$ & $8.25 \pm 3.56$ \\
\hline $\mathrm{t}$ & 7.9565 & 22.3663 & 5.4446 & 4.1985 \\
\hline $\mathrm{P}$ & 0.0000 & 0.0000 & 0.0000 & 0.0000 \\
\hline
\end{tabular}

\section{Comparative analysis of cardiac functional indexes between the two groups}

LVEFs of patients in the study group are significantly greater than those in the reference group $(\mathrm{P}<0.05)$, while LVESD and LVEDD are both smaller than those in the reference group $(\mathrm{P}<0.05)$, as shown in Table 3.

Table 3. Comparative analysis of cardiac functional indexes between the two groups $(\bar{x} \pm s)$.

\begin{tabular}{|c|c|c|c|c|}
\hline Group & & LVEF (\%) & LVESD (mm) & LVEDD (mm) \\
\hline $\begin{array}{l}\text { Study } \\
(n=49)\end{array}$ & group & $46.56 \pm 6.54$ & $46.25 \pm 5.02$ & $57.85 \pm 4.56$ \\
\hline $\begin{array}{l}\text { Reference } \\
(n=49)\end{array}$ & group & $40.03 \pm 7.02$ & $52.98 \pm 5.10$ & $62.06 \pm 4.26$ \\
\hline $\mathrm{t}$ & & 4.7642 & 6.5831 & 4.7225 \\
\hline
\end{tabular}




\section{$\begin{array}{llll}P & 0.0000 & 0.0000 & 0.0000\end{array}$ \\ Comparative analysis of occurrences of adverse reactions between the two groups}

Occurrence rate of adverse reactions in the study group is $10.20 \%$, which is insignificantly different from that in the reference group $(8.16 \%)(\mathrm{P}>0.05)$, as shown in Table 4 .

Table 4. Comparative analysis of occurrences of adverse reactions between the two groups $(n(\%))$.

\begin{tabular}{llll}
\hline Group & Emesis & Insomnia & Total occurrence rate \\
\hline Study group $(n=49)$ & $3(6.12)$ & $2(4.08)$ & $5(10.20)$ \\
\hline Reference group $(n=49)$ & $2(4.08)$ & $2(4.08)$ & $4(8.16)$ \\
\hline$X^{2}$ & & & 0.1223 \\
\hline$P$ & & 0.7265 \\
\hline
\end{tabular}

\section{Discussion}

Patients with coronary heart diseases suffer from dyslipidemia and enhanced blood viscosity of varying degrees, and blood viscosity gradually increases under continuous disease progression [5]. Statins lipid-lowering drugs are usually used in clinical treatment because these drugs can effectively lower the TC and LDL-C levels of patients, provide antiinflammation, antioxidation, and improvement effects of vascular endothelial functions, and inhibit the formation of atherosclerotic plaques [6]. Meanwhile, Tatins drugs can also lower peripheral arterial pressure; hence, these drugs have been extensively applied in the treatment of diseases, such as atherosclerosis and hypercholesterolemia [7].

Today, atorvastatin is a commonly used Tatins drug for the treatment of coronary heart diseases. As a common Tatins lipid-lowering drug, atorvastatin reduces cholesterol content in blood plasma by inhibiting synthesis of HMG-CoA reductase and cholesterol inside the liver, thereby facilitating LDL ingestion and catabolism and lowering LDL level by increasing quantity of LDL receptors on hepatocyte surface [8,9]. As a kind of piperazine derivative, trimetazidine can accelerate transformation of myocardial fatty acid metabolism in blood into glucose metabolism, inhibit generation of oxygen radicals, increase myocardial output quantity, stabilize intracellular environment, and improve myocardial functions [10]. The combined application of atorvastatin and trimetazidine in clinical treatment can fully provide effects of improving myocardial and vascular functions, maintaining long-term drug effect, and enhancing therapeutic effect.

The results of this study are as follows. (1) The total effective rate of patients in the study group is significantly higher than that in the reference group $(\mathrm{P}<0.05)$; (2) TG, TC, LDL-C, and HDL-C in the study group are all significantly lower than those in the reference group $(\mathrm{P}<0.05)$; (3) LVEFs of patients in the study group are significantly larger than those in the reference group $(\mathrm{P}<0.05)$, while LVESD and LVEDD are both significantly smaller than those in the reference group $(\mathrm{P}<0.05)$; (4) The occurrence rate of adverse reactions in the study group is insignificantly different from that in the reference group $(\mathrm{P}>0.05)$, all of which are quite similar to the related literature report. The obtained data from this study are as follows: (1) TG, TC, LDL-C, and HDL-C of patients in the observation group are $(1.36 \pm 0.28),(2.24 \pm 0.18),(0.75 \pm$ $0.03)$, and $(1.67 \pm 0.21 \mathrm{mmol} / \mathrm{L})$, respectively, which are significantly lower than those in the control group $((1.90 \pm$ $0.38),(3.29 \pm 0.23),(0.98 \pm 0.04)$, and $(1.94 \pm 0.27 \mathrm{mmol} / \mathrm{L})$, respectively) $(\mathrm{P}<0.05)$; (2) LVEFs of patients in the observation group are approximately $(46 \pm 9 \%)$, which are significantly lower than those in the control group (approximately $(40 \pm 8))(\mathrm{P}<0.05)$; (3) LVESD and LVEDD in the observation group are $(46 \pm 6$ and $58 \pm 4 \mathrm{~mm})$, respectively, which are significantly smaller than those in the reference group (approximately $(53 \pm 6$ and $62 \pm 4 \mathrm{~mm})$, respectively) $(\mathrm{P}<0.05)$; (4) The occurrence rate of adverse reactions in the observation group is $12.5 \%$ while that in the control group is $10.0 \%$. Hence, no significant difference exists between the two group $(\mathrm{P}>0.05)$. Therefore, these findings indicate that curative effect of atorvastatin-trimetazidine combined treatment of coronary heart diseases is excellent.

\section{Conclusion}

Overall, atorvastatin-trimetazidine combined treatment of patients with coronary heart diseases has a significant clinical effect and can effectively improve the blood lipid levels and cardiac functions of patients. Therefore, such combination is a therapeutic schedule worthy of clinical promotion and application with favorable safety and reliability.

\section{References}

1. Lin X, Ma A, Zhang W, Lu Q, Sun C, Tian H, Lei X, Bai $\mathrm{X}$. Cardioprotective effects of atorvastatin plus trimetazidine in percutaneous coronary intervention. Pak J Med Sci 2013; 29: 545-548.

2. Senthil KTK, Ganesh EN, Umamaheswari R. Lung nodule volume growth analysis and visualization through autocluster k-means segmentation and centroid/shape variance based false nodule elimination. Biomed Res India 2017; 28: 1927-1934.

3. Malekinejad H, Shafie-Irannejad V, Hobbenaghi R, Tabatabaie SH, Moshtaghion SM. Comparative protective effect of hawthorn berry hydroalcoholic extract, atorvastatin, and mesalamine on experimentally induced colitis in rats. J Med Food 2013; 16: 593-601.

4. Zhang J, Li P, Wang X. Beneficial effect of xanthohumol, a prenylated flavanoid, on 3-nitropropionic acid induced huntingtons disease in a rat model. Lat Am J Pharm 2017; 36: 1867-1874.

5. Sandhiya S, Dkhar SA, Pillai AA, George M, Jayaraman B, Chandrasekaran A. Comparison of ranolazine and trimetazidine on glycemic status in diabetic patients with coronary artery disease-a randomized controlled trial. J Clin Diagn Res 2015; 9: 1-5. 
6. Zhu GF. Exposure of bisphenol A (BPA) derivatives affect the metabolic elimination of Alzheimers diseases treatment drugs. Lat Am J Pharm 2017; 36: 1753-1759.

7. Masic I, Rahimic M, Dilic M, Kadribasic R, Toromanovic S. Socio-medical characteristics of coronary disease in Bosnia and Herzegovina and the world. Mater Sociomed 2011; 23: 171-183.

8. Berry C, Corcoran D, Hennigan B, Watkins S, Layland J, Oldroyd KG. Fractional flow reserve-guided management in stable coronary disease and acute myocardial infarction: recent developments. Eur Heart J 2015; 36: 3155-3164.

9. Kalem F, Durmaz S, Ozdemir B, Ergun AG, Ertugrul O. The diagnostic value of procalcitonin, WBC, and CRP in diagnosis of lower respiratory tract infections in elderly patients. Biomed Res India 2017; 28: 1012-1015.
10. Blais C, Rochette L. Trends in prevalence, incidence and mortality of diagnosed and silent coronary heart disease in Quebec. Health Promot Chronic Dis Prev Can 2015; 35: 184-193.

\section{*Correspondence to}

Li-Li Wang

Department of the First Heart Surgery

Tangshan Workers' Hospital

PR China 